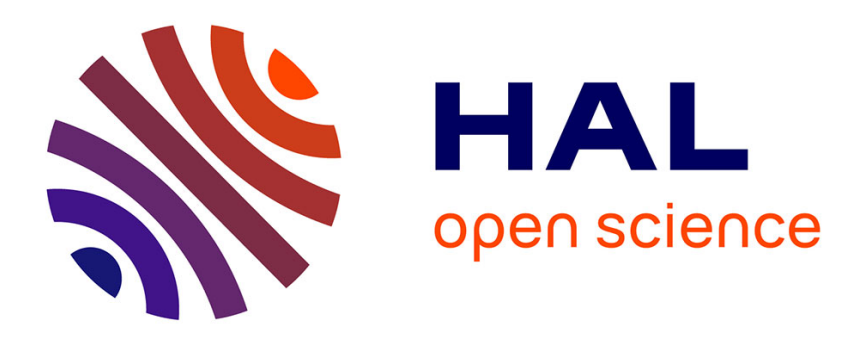

\title{
Détection de spécialistes de la taille de la pierre au Rubané
}

\author{
Pierre Allard
}

\section{To cite this version:}

Pierre Allard. Détection de spécialistes de la taille de la pierre au Rubané. Bulletin de la Société préhistorique française, 2012, 109 (2), pp.267-278. 10.3406/bspf.2012.14107 . hal-01476229

\author{
HAL Id: hal-01476229 \\ https://hal.science/hal-01476229
}

Submitted on 21 Mar 2017

HAL is a multi-disciplinary open access archive for the deposit and dissemination of scientific research documents, whether they are published or not. The documents may come from teaching and research institutions in France or abroad, or from public or private research centers.
L'archive ouverte pluridisciplinaire HAL, est destinée au dépôt et à la diffusion de documents scientifiques de niveau recherche, publiés ou non, émanant des établissements d'enseignement et de recherche français ou étrangers, des laboratoires publics ou privés.

\section{(ㅇ)(1) $\$$}

Distributed under a Creative Commons Attribution - NonCommercial - NoDerivatives $\mid 4.0$ 


\section{Détection de spécialistes de la taille de la pierre au Rubané} Pierre Allard

\section{Citer ce document / Cite this document :}

Allard Pierre. Détection de spécialistes de la taille de la pierre au Rubané. In: Bulletin de la Société préhistorique française, tome $109, \mathrm{n}^{\circ} 2,2012$. pp. 267-278;

doi : 10.3406/bspf.2012.14107

http://www.persee.fr/doc/bspf_0249-7638_2012_num_109_2_14107

Document généré le 08/03/2017 


\begin{abstract}
The matter of this paper is about the Linear Pottery Culture, an Early Neolithic culture in Central Europe. The richly documented assemblages allow demonstrating that the lithic productions are technically rather simple and more particularly with regard to the common toolkit used by everyone. Thus, the main part of the common toolkit is composed of several types of tools made on unretouched or truncated blades serving as blanks. Debitage is oriented towards the production of small-sized blades obtained by indirect percussion which are attested in all the artifactual household assemblages. Despite this apparent simplicity, a more complex level can be stated within the general operational sequence of flint knapping concerning notably the procurement of lithic raw materials. Henceforth, it is a wellknown fact that the area of flint procurement of these groups reaches far beyond their local environment and rather aims at the procurement of mostly regional, even exogenous raw materials of good quality. It is a fact that from the very start, these groups use items in their daily life they have to acquire from other groups (this is frequently the case for adzes or ornaments, for example). From then on, flint mines and important extraction places are known from outside the settlements. They are thought to represent an initial step in the process of specialisation of the knapping techniques. It appears indeed that the processes of procurement and distribution of flint products are more precisely suggestive of possible specialisation of flint knapping. As a matter of fact, it can be demonstrated that distinct, extradomestic blade productions raise the question of the existence of skilled knappers devoted to intensive production of standardized laminar products that circulate within more or less large-scale diffusion networks. The phenomenon is interesting as it seemingly emerges at a distinct moment within the development of the Linear Pottery Culture and as it concerns several regions with rich flint raw material sources. Moreover, the question may be raised whether this economical phenomenon had direct incidence on the development of efficient variants of debitage as they are known from the Late Linear Pottery Culture at Verlaine in Belgium for example. Nonetheless, if, according to the different components, it is possible to consider these intensive productions of flint blades as productions with a high level of specialisation, the question of genuine specialists of flint knapping has to be taken into account not only with regard to the invested know-how but also with regard to the objectives of the production. It comes out, however, that, though the skill of the flintknappers is clearly high in distinct cases, the production made by these skilled individuals does not show any difference compared to the classical production that can be found everywhere, in almost all settlements and household assemblages. This is why we put forward here the distinction between specialised production and production made by a specialist (Translation : Karoline Mazurié de Keroualin).
\end{abstract}

\title{
Résumé
}

Nous traiterons ici du Rubané, une culture correspondant au Néolithique ancien de l'Europe tempérée. La richesse de la documentation permet de montrer que les productions lithiques sont techniquement assez simples et particulièrement en ce qui concerne le fonds commun partagé par tous. Ainsi, l'essentiel de l'outillage est composé de quelques catégories d'outils sur supports laminaires bruts ou tronqués et le débitage est orienté vers des lames de petits modules réalisées à l'aide de la percussion indirecte dont on retrouve les témoins dans toutes les maisonnées. Malgré cette simplicité apparente, on peut relever dans la chaîne opératoire générale de l'activité de taille un degré plus complexe en ce qui concerne l'acquisition des matériaux. II est bien connu désormais que le territoire d'acquisition des silex de ces populations dépasse très largement l'environnement local et est plutôt orienté vers l'acquisition de matières premières de bonne qualité, le plus souvent régionales ou exogènes. C'est un fait que ces populations intègrent dans leur quotidien d'emblée des objets qu'elles doivent acquérir auprès d'autres communautés (souvent le cas des herminettes ou de la parure par exemple). Pour le silex, on connaît désormais des minières et des lieux d'extraction importants déconnectés des habitats et qui pourraient révéler un premier degré de spécialisation des activités de taille. II semblerait en effet que les processus d'acquisition et de distribution des produits siliceux soient les éléments qui suggèrent de s'interroger sur la spécialisation éventuelle de l'activité de taille. En effet, on peut démontrer que certaines des productions laminaires effectuées hors du cadre domestique posent, dans la 
production intensive de produits laminaires normés qui sont mis en circulation dans des réseaux à plus ou moins large échelle de diffusion. Le phénomène est intéressant car il semble se mettre en place à un certain moment dans la séquence du Rubané et concerne plusieurs régions riches en matières premières siliceuses. On peut d'ailleurs s'interroger pour savoir si ce phénomène économique n'a pas eu une incidence directe sur la mise en place de variantes de débitage efficaces, telles qu'on les connaît au Rubané récent à Verlaine en Belgique par exemple. Néanmoins, ces productions intensives de lames en silex peuvent être considérées, d'après les différentes composantes, comme des productions qui présentent un degré de spécialisation évident, la question des tailleurs réellement spécialistes doit être envisagée non seulement au niveau des savoir-faire engagés mais également au niveau des objectifs de production. Or il s'avère que si la compétence des tailleurs dans certains cas est clairement élevée, la production réalisée par ces tailleurs expérimentés ne montre aucune différence avec la production classique que l'on retrouve partout, aussi bien dans tous les villages que dans toutes les maisonnées. C'est pourquoi nous proposerons ici de faire une distinction entre production spécialisée et production de spécialiste. 


\title{
Pierre ALLARD au Rubané
}

\begin{abstract}
Résumé
Nous traiterons ici du Rubané, une culture correspondant au Néolithique ancien de l'Europe tempérée. La richesse de la documentation permet de montrer que les productions lithiques sont techniquement assez simples et particulièrement en ce qui concerne le fonds commun partagé par tous. Ainsi, l'essentiel de l'outillage est composé de quelques catégories d'outils sur supports laminaires bruts ou tronqués et le débitage est orienté vers des lames de petits modules réalisées à l'aide de la percussion indirecte dont on retrouve les témoins dans toutes les maisonnées.

Malgré cette simplicité apparente, on peut relever dans la chaîne opératoire générale de l'activité de taille un degré plus complexe en ce qui concerne l'acquisition des matériaux. Il est bien connu désormais que le territoire d'acquisition des silex de ces populations dépasse très largement l'environnement local et est plutôt orienté vers l'acquisition de matières premières de bonne qualité, le plus souvent régionales ou exogènes. C'est un fait que ces populations intègrent dans leur quotidien d'emblée des objets qu'elles doivent acquérir auprès d'autres communautés (souvent le cas des herminettes ou de la parure par exemple). Pour le silex, on connaît désormais des minières et des lieux d'extraction importants déconnectés des habitats et qui pourraient révéler un premier degré de spécialisation des activités de taille.

Il semblerait en effet que les processus d'acquisition et de distribution des produits siliceux soient les éléments qui suggèrent de s'interroger sur la spécialisation éventuelle de l'activité de taille. En effet, on peut démontrer que certaines des productions laminaires effectuées hors du cadre domestique posent, dans la plupart des cas, des questions concernant l'existence de tailleurs spécialistes voués à une production intensive de produits laminaires normés qui sont mis en circulation dans des réseaux à plus ou moins large échelle de diffusion. Le phénomène est intéressant car il semble se mettre en place à un certain moment dans la séquence du Rubané et concerne plusieurs régions riches en matières premières siliceuses. On peut d'ailleurs s'interroger pour savoir si ce phénomène économique n'a pas eu une incidence directe sur la mise en place de variantes de débitage efficaces, telles qu'on les connaît au Rubané récent à Verlaine en Belgique par exemple.

Néanmoins, ces productions intensives de lames en silex peuvent être considérées, d'après les différentes composantes, comme des productions qui présentent un degré de spécialisation évident, la question des tailleurs réellement spécialistes doit être envisagée non seulement au niveau des savoir-faire engagés mais également au niveau des objectifs de production. Or il s'avère que si la compétence des tailleurs dans certains cas est clairement élevée, la production réalisée par ces tailleurs expérimentés ne montre aucune différence avec la production classique que l'on retrouve
\end{abstract}


partout, aussi bien dans tous les villages que dans toutes les maisonnées. C'est pourquoi nous proposerons ici de faire une distinction entre production spécialisée et production de spécialiste.

\section{Mots clefs}

Néolithique ancien, industrie lithique, spécialisation, production spécialisée, production domestique.

\section{Abstract}

The matter of this paper is about the Linear Pottery Culture, an Early Neolithic culture in Central Europe. The richly documented assemblages allow demonstrating that the lithic productions are technically rather simple and more particularly with regard to the common toolkit used by everyone. Thus, the main part of the common toolkit is composed of several types of tools made on unretouched or truncated blades serving as blanks. Debitage is oriented towards the production of small-sized blades obtained by indirect percussion which are attested in all the artifactual household assemblages.

Despite this apparent simplicity, a more complex level can be stated within the general operational sequence of fint knapping concerning notably the procurement of lithic raw materials. Henceforth, it is a wellknown fact that the area of flint procurement of these groups reaches far beyond their local environment and rather aims at the procurement of mostly regional, even exogenous raw materials of good quality. It is a fact that from the very start, these groups use items in their daily life they have to acquire from other groups (this is frequently the case for adzes or ornaments, for example). From then on, flint mines and important extraction places are known from outside the settlements. They are thought to represent an initial step in the process of specialisation of the knapping techniques.

It appears indeed that the processes of procurement and distribution of flint products are more precisely suggestive of possible specialisation of flint knapping. As a matter of fact, it can be demonstrated that distinct, extra-domestic blade productions raise the question of the existence of skilled knappers devoted to intensive production of standardized laminar products that circulate within more or less large-scale diffusion networks. The phenomenon is interesting as it seemingly emerges at a distinct moment within the development of the Linear Pottery Culture and as it concerns several regions with rich flint raw material sources. Moreover, the question may be raised whether this economical phenomenon had direct incidence on the development of efficient variants of debitage as they are known from the Late Linear Pottery Culture at Verlaine in Belgium for example.

Nonetheless, if, according to the different components, it is possible to consider these intensive productions of flint blades as productions with a high level of specialisation, the question of genuine specialists of flint knapping has to be taken into account not only with regard to the invested know-how but also with regard to the objectives of the production. It comes out, however, that, though the skill of the flintknappers is clearly high in distinct cases, the production made by these skilled individuals does not show any difference compared to the classical production that can be found everywhere, in almost all settlements and household assemblages. This is why we put forward here the distinction between specialised production and production made by a specialist (Translation: Karoline Mazurié de Keroualin).

\section{Keywords}

Early Neolithic, lithic industry, specialization, territorial analysis, specialized production, household production.

Nous traiterons ici du Rubané, une culture correspondant au Néolithique ancien de l'Europe tempérée. Cette entité qui connaît une extension remarquable à l'échelle de l'Europe dans la seconde moitié du
$\mathrm{VI}^{\mathrm{e}}$ millénaire avant notre ère présente dans sa culture matérielle un fonds commun partagé qui montre une certaine homogénéité de ses productions et de son architecture. 
Néanmoins, au delà de cette production globalement homogène, l'approche techno-économique et fonctionnelle des mobiliers lithiques nous permet de présenter différents cas de figures qui posent deux questions principales :

- peut-on démontrer l'existence de productions spécialisées pour le Néolithique ancien rubané?

- et ces éventuelles productions sont elles le fruit de spécialistes indépendants de la structure domestique au sens large?

Nous entendons ici par espace domestique un lieu de vie et d'activités diverses plus ou moins élaborées.

\section{LE MATÉRIEL LITHIQUE TAILLÉ AU RUBANÉ, PRÉSENTATION GÉNÉRALE}

Prise dans sa globalité, l'industrie lithique taillée des premières populations agro-pastorales de l'Europe tempérée peut être perçue comme relativement normée et bien que l'aire d'expansion du Rubané soit considérable, la diversité apparaît plutôt faible.

En effet, la chaîne opératoire de la taille du silex est assez homogène et comporte des similarités fortes entre chaque région à différents niveaux, que ce soit dans le territoire d'acquisition des ressources (très souvent diversifié au Rubané), la production laminaire ou encore la panoplie de l'outillage. La production laminaire est largement majoritaire à de rares exceptions près et il s'agit exclusivement d'un débitage réalisé à la percussion indirecte de petites lames $(6-10 \mathrm{~cm}$ de longueur en moyenne). Ces faibles modules, totalement indépendants des ressources siliceuses, ont été reconnus comme un véritable marqueur culturel fort de cette industrie et si l'on peut discerner des variantes dans la gestion des débitages, l'objectif des productions est largement partagé par ces populations. Un même constat peut être fait à propos de la panoplie de l'outillage qui est composée essentiellement de grattoirs, faucilles et perçoirs et de lames à retouches variables. Le tool-kit est peu diversifié et assez monotone. On a également observé que la plupart des outils sont utilisés sur des supports $a d$ hoc, révélant ainsi une grande simplicité dans la gestion des supports et dans l'aménagement des outils (Allard, 2005).

Les productions annexes sont rares et concernent essentiellement des productions expédientes d'éclats qui sont utilisés bruts et rejetés rapidement (par exemple le débitage de galet en quartz: Mauvilly, 1997).

Il faut remarquer une assez bonne homogénéité des formes typologiques récurrentes qui se retrouvent parfois de la Pologne à l'Atlantique comme les perçoirs sur lame à double bords abattus convergent ou encore les armatures de flèche qui, si elle connaissent une évolution, ne comptent guère qu'un ou deux modèles contemporains.

Cette industrie peut donc se définir comme simple étant donné que les principaux caractères qui la déterminent ne sont pas très élaborés (Allard et al., 2007) : percussion indirecte ordinaire, petit module laminaire, outillage peu investi (la technique du microburin ou encore la retouche rasante apicale des pointes de flèche symétriques apparaissent comme les éléments les plus investis). La composante laminaire est le dénominateur commun et peut être considérée comme l'objectif principal de la taille du silex par ces populations, au regard de la panoplie d'outils.

La diversité existe néanmoins et se manifeste essentiellement dans la présence ou l'absence de certains types d'outils ou de rares singularités typologiques (comme les «quartiers d'orange» du Rubané de Hesbaye ou encore les burins du Rubané du bassin de la Seine). Il faut également remarquer que si certains types d'outils caractérisent les assemblages lithiques régionaux, les analyses tracéologiques montrent que les stigmates fonctionnels de ces outils singuliers existent en fait sur d'autres types d'outils ou de supports. C'est le cas du «poli $23 »$ recensé par exemple sur les quartiers d'orange de Hesbaye ou sur les burins du Rubané du bassin de la Seine, mais aussi sur de nombreux produits bruts ou retouchés différents (Caspar, 1988; Van Gijn, 1990; Vaughan, 1994; Allard et al., 2004).

\section{L'ACQUISITION DES MATÉRIAUX ET LA SURPRODUCTION}

Malgré cette simplicité apparente, un degré de complexité évident peut être observé dans l'industrie lithique en ce qui concerne le territoire d'acquisition des matières premières. En effet, les travaux menés sur l'approvisionnement en silex de ces communautés sont concordants sur plusieurs points qui caractérisent la gestion des ressources siliceuses. Contrairement à une première idée reçue, ces populations utilisent fréquemment des matériaux tous azimuts et d'une bonne qualité pour le débitage des lames. Le cas le plus fréquent correspond à des matériaux régionaux $(20-40 \mathrm{~km})$ qui sont acheminés dans le village déjà mis en forme et débités in situ dans chaque maisonnée. En effet, l'implantation des hameaux est principalement orientée vers les terrains agricoles et il ne semble pas que les ressources siliceuses soient un facteur déterminant pris en compte par ces populations. De plus, on a pu démontrer que dans les sources d'approvisionnement des différents types de matériaux (silex, roche tenace, parure par exemple), la part des gîtes régionaux est majoritaire et les sources exogènes quasi-systématiques (Bakels, 1982), ce qui renforce l'idée que les ressources lithiques participent peu du choix du lieu d'installation des hameaux.

Pour en reprendre l'historique, les chercheurs polonais ont très tôt mis en évidence que l'acquisition des silex était en fait un système complexe construit sur des relations socio-économiques entre les occupations où l'on peut distinguer différents maillons, du site d'extraction (à notre connaissance toujours déconnecté de l'habitat stricto sensu) au site redistributeur vers des sites consommateurs. Ces circulations de silex, sous diverses formes (nucléus et lames) sont donc organisées en véritables réseaux qui distribuent des produits sur de larges distances, reliant les grandes aires 


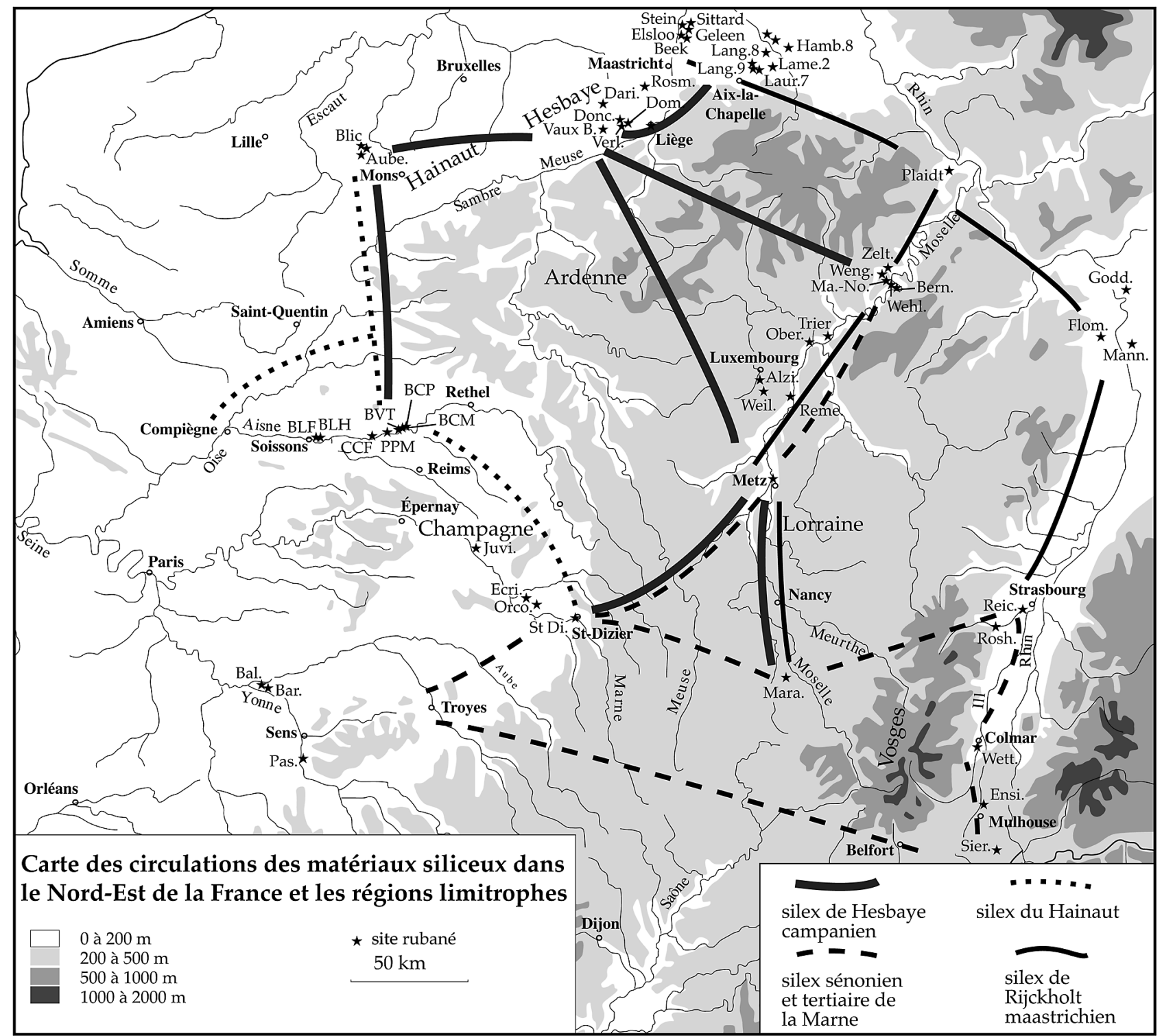

Fig. 1 - Carte des circulations de silex au Rubané récent (Allard, 2005). Fig. 1 - Distribution map of flint networks in the late Bandceramic.

d'implantation des habitats sur des centaines de kilomètres (fig. 1). Par ailleurs, des petites quantités de silex circulent également qui sont plutôt interprétées comme les témoins de contacts entre les différentes communautés villageoises (Lech 1987 et 1990).

Un degré de complexité supplémentaire a été proposé sur le plateau de Langweiller d'après la répartition différentielle des déchets de taille dans les différents sites de cette micro-région. En effet, le premier habitat qui occupe le plateau (Langweiller 8) est également le seul a avoir une durée d'occupation continue pour l'ensemble de la séquence. Le mobilier lithique montre que les déchets du débitage laminaire sont essentiellement concentrés dans cet habitat et il est considéré comme un lieu de redistribution des produits en silex (place centrale) vers les autres occupations, l'idée sous jacente est que son ancienneté lui aurait permis un certain contrôle des gites de matières premières par le biais de relations socio-économiques (Zimmermann, 1995).

La découverte en contexte d'occupations domestiques d'amas de déchets de débitage en grande quantité a plus clairement posé la question d'une économie de surproduction par rapport aux besoins villageois. C'est un phénomène bien attesté dans plusieurs régions en Europe occidentale comme à Verlaine en Hesbaye liégeoise (Burnez-Lanotte et Allard, 2003), à Beek Kerkeveld dans le Limbourg en Hollande (De Grooth, 1987) ou encore à en Allemagne à Hanau Klein Auheim (Sommer, 2006). Il faut garder en mémoire que les sites de la Céramique Linéaire sont fortement érodés et que cette documentation est donc probablement minime par rapport à la réalité historique de ces productions intensives. Dans ces sites, on retrouve des amas de déchets du débitage, la plupart du temps secondairement rejetés dans des structures, et qui sont les vestiges d'une 
production laminaire massive. À Verlaine, ce sont des dizaines de milliers de déchets et des milliers de nucléus qui ont été retrouvés. C'est donc clairement avec cette documentation qu'il y a matière à réfléchir pour aborder le problème de la spécialisation des tailleurs et/ou des productions pour le Rubané. Afin de développer cette réflexion, nous allons d'une part décrire la production qui est issue de ces amas de débitage et d'autre part apporter des précisions sur le savoir-faire des tailleurs.

\section{LES AMAS DE DÉBITAGE DE LAMES EN SILEX DU RUBANÉ}

Concernant les productions, les amas de silex de Hesbaye et de Beek sont principalement, voire exclusivement les vestiges du débitage de lames en silex. Seul Darion se distingue par la présence importante du débitage d'éclats par la tranche dans les amas (Jadin, 1990), phénomène que nous n'avons pas repéré dans les ensembles de Verlaine, Donceel et Dommartin, et qui semble également absent à Beek (De Grooth, 1987).

Cette production de lames lorsqu'elle est caractérisée, ne semble pas du tout distincte de la production laminaire habituelle. En effet, elle est orientée vers des calibres communs de la période, c'est-à-dire des produits dont la longueur oscille entre 8 et $12 \mathrm{~cm}$ de long pour un intervalle large. Cette observation est particulièrement spectaculaire sur le site de Verlaine puisque sur les centaines de nucléus retrouvés intacts (non réemployés), un sur deux est abandonné entre 9,5 et $11 \mathrm{~cm}$ de long, et $85 \%$ entre 8 et $10 \mathrm{~cm}$ de long, information validée par la mesure des derniers négatifs des lames sur les surfaces laminaires (76\% entre 8 et 11,5 cm de long).

Ces amas sont également composés de matériaux qui participent aux réseaux d'approvisionnement à plus ou moins longue distance (c'est paradoxalement les distances les plus courtes qui sont délicates à mettre en évidence). En effet, on distingue des distances de circulations de l'ordre de $200 \mathrm{~km}$ pour les silex de Rijckholt et de Hesbaye. Les sites «consommateurs de ces produits » permettent par ailleurs de montrer que la norme métrique est respectée et les procédés de détachement des lames mis en évidence dans les amas sont retrouvés sur les produits exogènes des sites consommateurs, ce qui démontre qu'il s'agit bien des produits issus des régions de productions massives (Allard, 2005).

D'un point de vue spatial, les découvertes concordent pour affirmer que cette production intensive est déconnectée de la production domestique (nous entendons production domestique celle représentée par les vestiges retrouvés dans les couches détritiques des fosses latérales des unités d'habitation). Par exemple, les amas de silex de Darion ne sont pas retrouvés dans les fosses latérales des unités d'habitation, mais dans des structures diverses hors de l'espace domestique. À Verlaine, cette situation est la même à de rares exceptions près. Alors que la plupart des amas sont retrouvés hors des fosses liées aux unités d'habitation, trois amas étaient présents dans les fosses latérales de deux maisons accolées. L'étude fine de la distribution des vestiges dans la stratigraphie de ces structures a permis de monter qu'il s'agit d'un type de rejet court dans le temps et qui ne correspond pas à celui des couches détritiques des fosses latérales (Allard et BurnezLanotte, à paraître). Par ailleurs, le calcul de productivité entrepris sur cet habitat pour donner un ordre de grandeur de la production laminaire a bien montré que le mobilier de la couche détritique était a priori complet et que la production des amas de cette même structure apparaît très largement surnuméraire et surtout avec un très grand déficit des produits laminaires (Allard et Burnez-Lanotte, 2008).

Néanmoins, il faut retenir que la composition de ces amas ne diffère par ailleurs qu'assez peu de celle des couches détritiques domestiques (Allard, 2005). Cela oppose des ensembles composés de déchets bruts du débitage dans les amas à des ensembles détritiques des fosses latérales composés d'outils, de rebus, d'objets brûlés, mais également de déchets de taille attestant de l'ensemble des étapes de la chaîne opératoire du débitage des lames.

Un critère quantitatif peut être avancé pour caractériser ces productions. Le volume de mobilier issu de ces amas est considérable en comparaison avec ce que l'on retrouve communément dans les habitats de la période. En prenant l'exemple de la Hesbaye, on pourrait postuler qu'il s'agit d'un des quelques cas en Europe où coexiste un environnement favorable à l'implantation des villages et des ressources siliceuses aisément disponibles et de bonne qualité. Le silex est abondant et les séries lithiques sont de ce fait fournies par rapport aux habitats implantés dans des régions pauvres en ressources siliceuses locales. Néanmoins, les amas de silex ne sont pas retrouvés dans tous les habitats et sont jusqu'à présent l'apanage de certains sites ou de certaines microrégions. Les effectifs sont alors considérables, des dizaines de milliers d'objets qui révèlent l'exploitation de milliers de blocs en silex pour l'obtention de supports laminaires normés. Le phénomène de surproduction n'est plus à démontrer dans ce cas et les estimations réalisées à partir des remontages du site de Verlaine montrent qu'il s'agit de dizaines de milliers de lames qui ont été débitées puis mises en circulation dans des réseaux de distribution de produits siliceux (Allard et Burnez-Lanotte, 2008).

En résumé, on peut retenir que la production laminaire dont témoignent les amas est similaire à la production domestique. En effet, il s'agit des mêmes modules et l'emploi des produits sur les sites consommateurs ne révèle pas de différence fonctionnelle pour les silex exogènes, du moins d'après les inventaires des catégories typologiques reconnues sur ces habitats. Il s'agit en revanche d'une production que l'on peut qualifier d'intensive à cause des quantités estimées d'après les déchets retrouvés dans ces amas et, de plus, elle se distingue spatialement la plupart du temps des structures qui nous livrent les ensembles détritiques domestiques. 


\section{LES DIFFÉRENTES ÉCHELLES D'OBSERVATION QUALITATIVES DES PRODUCTIONS LAMINAIRES}

Le second aspect de ces productions massives concerne les méthodes, les savoir-faire et les niveaux de compétence engagés. Il faut reconnaître le manque de données contextuelles, faute d'études systématiques et de remontages dans la plupart des contextes de productions intensifs des produits laminaires. Les informations disponibles sont principalement issues des ensembles remontés ou partiellement remontés de Beek, Liège et de Verlaine.

Concernant la méthode de taille laminaire, on peut mettre en évidence des différences qualitatives dans les débitages réalisés qui sont de différents degrés selon les échelles d'observation prises en compte. En effet, on proposera ici plusieurs niveaux, le premier concerne le niveau de compétence reconnu dans les amas, le second entre les amas et enfin, le dernier, d'un point de vue plus général puisqu'il existe des variantes du débitage des lames pour cette période.

\section{La compétence des tailleurs rubanés : le cas des amas de Verlaine}

Le propos est ici d'illustrer le savoir-faire mis en oeuvre concernant le débitage des productions laminaires normées. Nous avons choisi de prendre le cas de Verlaine puisque ce site offre une documentation abondante ainsi que des remontages poussés qui permettent d'appréhender correctement le niveau de compétence des tailleurs rubanés. À Verlaine, les tailleurs rubanés ont débités des produits laminaires en très grande quantité et ils ont adaptés de manière originale la méthode de taille, notamment sur les grands blocs de silex (Allard, 2007). Les remontages réalisés de l'amas 130 (quatre-vingt-quatre blocs de silex partiellement remontés, soit $29 \%$ en nombre et presque $76 \%$ en poids des 4272 pièces) ont permis d'évaluer les connaissances des tailleurs. L'examen des remontages démontre clairement que ce sont des bons tailleurs qui réalisaient les débitages puisque l'on observe une gestion pertinente des modalités d'aménagement et d'entretien des nucléus qui ont permis une production maximale en fonction des différents volumes (fig. 2). Les contraintes inhérentes de la matière première sont également contournées par des procédés adaptés et raisonnés. De même, aucune tentative de poursuite du débitage n'a été réalisée sur des blocs impropres à la taille ou présentant un défaut majeur en cours d'aménagement. Nous ne reviendrons pas ici sur le détail car cela a été publié par ailleurs (Allard, 2007), mais il faut retenir que plutôt que de sacrifier une partie des volumes, les blocs ont été taillés par la mise en place successive de surfaces laminaires tous azimuts afin de réaliser une production supplémentaire. On observe donc des blocs exploités au maximum selon des schémas complexes et astucieux avec l'alternance de préparation, plein débitage, d'abandon d'une surface laminaire pour recommencer la préparation d'une nouvelle surface laminaire. Les grands blocs de Verlaine (longueur supérieure à $20 \mathrm{~cm}$ ) montrent jusqu'à quatre installations successives de surface laminaires distinctes respectant toujours la norme dimensionnelle des lames de la période. Il faut donc constater le refus manifeste de produire des grandes lames (supérieures à $15 \mathrm{~cm}$ ), et la volonté de configurer les volumes afin d'obtenir une production optimale de produits calibrés de 8-12 cm. L'excellente qualité du débitage illustrée par ces schémas complexes se constate aussi par la gestion raisonnée des blocs de plus petite taille. En effet, la plupart des blocs montrent des débitages parfaitement exécutés, le plus souvent d'un seul tenant après l'ouverture du plan de frappe, sans aucune séquence d'entretien discernable.

Sans aucun doute possible, les tailleurs des amas de Verlaine étaient expérimentés et d'un bon niveau. Il faut également mentionner à Verlaine quelques cas de nucléus de très grandes dimensions qui suggèrent l'existence d'un débitage «hors norme». Il s'agit de deux pièces issues de la structure 01 dont nous avons étudié plus de 400 nucléus à lames et près de 25000 déchets de taille. La première est un nucléus dont le débitage a été exhaustif et dont on observe une série d'au moins trois lames qui ont été débitées sur toute la longueur avant une petite série de lames courtes réalisées en fin de débitage. La longueur des lames est légèrement inférieure à $17 \mathrm{~cm}$. Le nucléus est épuisé, son épaisseur est de l'ordre de $1,5 \mathrm{~cm}$. L'autre est un remontage d'une base fracturée en cours de débitage et d'un nucléus arrêté suite à un accident de taille. L'état initial bien visible sur ce raccord montre une préparation triangulaire à triple crête bien exécutée sur une longueur supérieure à $20 \mathrm{~cm}$ (fig. 3).

La rareté des remontages ne permet pas de développer dans le détail cette comparaison mais on peut remarquer que le degré du savoir-faire des amas de Verlaine est supérieur à celui qui transparaît des blocs remontés de l'amas rubané de Liège «Place SaintLambert». En effet, les comparaisons des procédés ou des modalités techniques pour le débitage laminaire entre l'amas de la couche 4 fosse 9 de Liège (Cahen, 1984) et les amas 130, 01 et 56 du site de Verlaine indiquent clairement des différences qualitatives dans les préparations des blocs ou dans la conduite du débitage des lames. Dans l'amas de Liège, le mobilier retranscrit bien la méthode du débitage laminaire dans son expression commune pour les ensembles de Hesbaye. Ces remontages montrent des préparations simples où les blocs sont mis en forme au plus près du gabarit des lames souhaitées. Les blocs sont d'ailleurs souvent amputés d'une grande partie de leur volume d'origine lors de l'ouverture du plan de frappe.

À Verlaine, les préparations à double ou triple crête, les nucléus à tables multiples, les grands volumes laminaires et les variantes du schéma opératoire de taille apparaissaient comme des éléments qui distinguent un degré de compétence plus élevé. 


\section{Un niveau suprarégional avec nouvelle méthode de taille}

En résumant les principales caractéristiques du débitage laminaire du Rubané, il faut constater qu'il existe des variantes dans le débitage des blocs de silex. Le débitage principal et ubiquiste des communautés danubiennes est laminaire par percussion indirecte. Cependant, au sein de cette production homogène, il existe plusieurs méthodes de la taille des lames en silex qui distinguent par exemple le Rubané rhéno-mosan de celui du Bassin parisien. En entrant dans le détail des
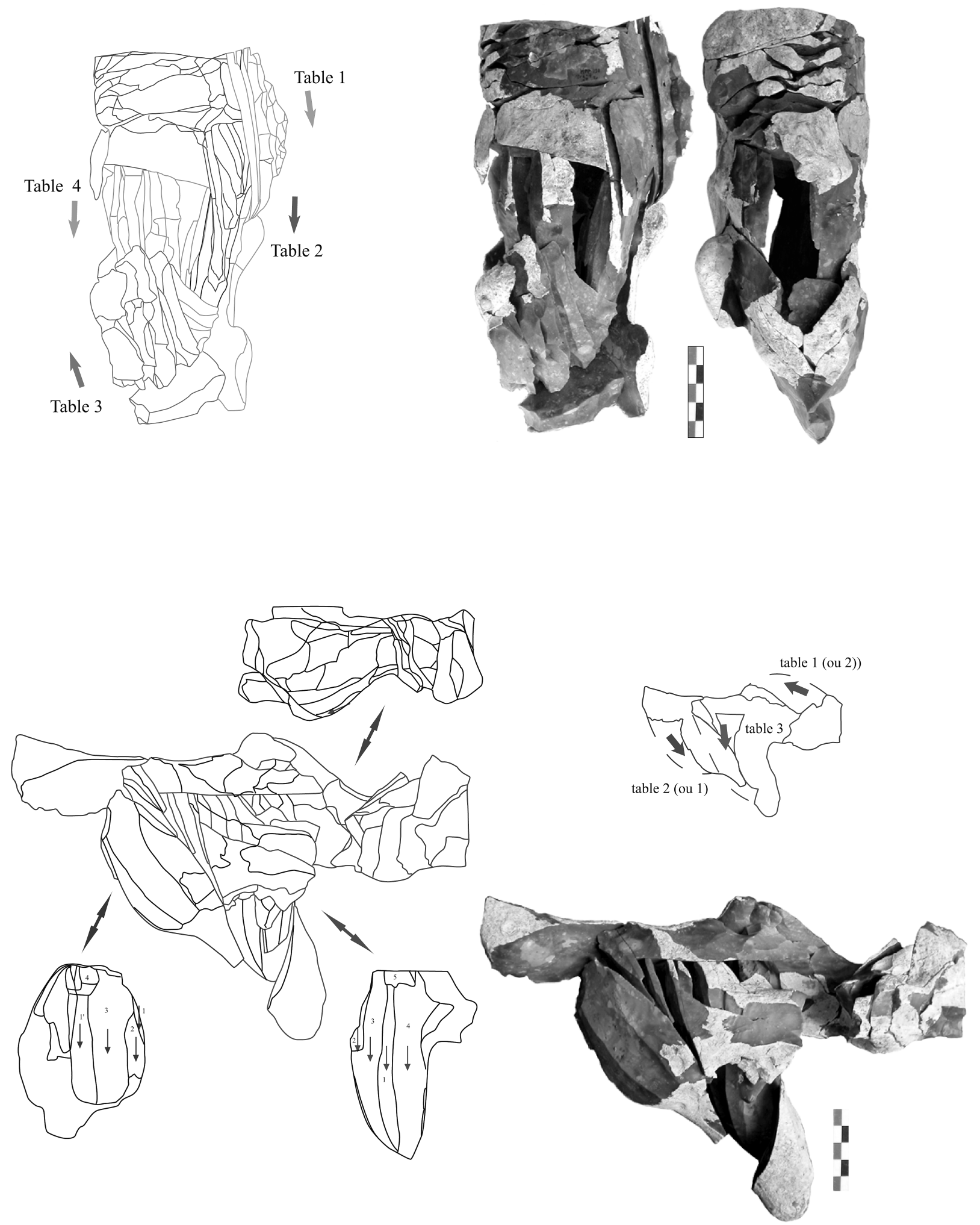

Fig. 2 - Remontages de l'amas de la st. 130 de Verlaine (Allard, 2007).

Fig. 2 - Reffiting of the debitage concentration in feature 130 of Verlaine (Allard, 2007). 
nucléus 50, st. 01, Verlaine
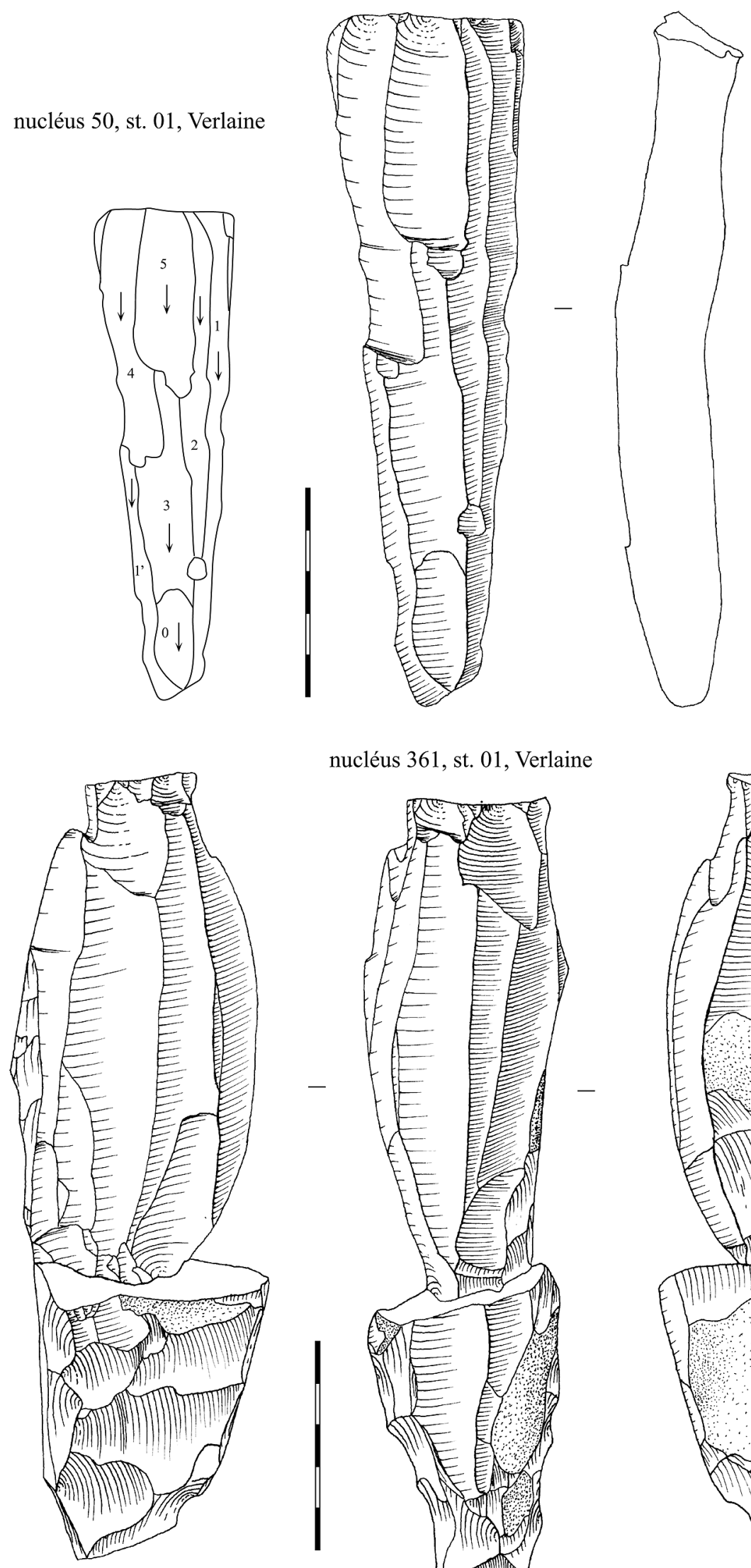

nucléus 361, st. 01, Verlaine
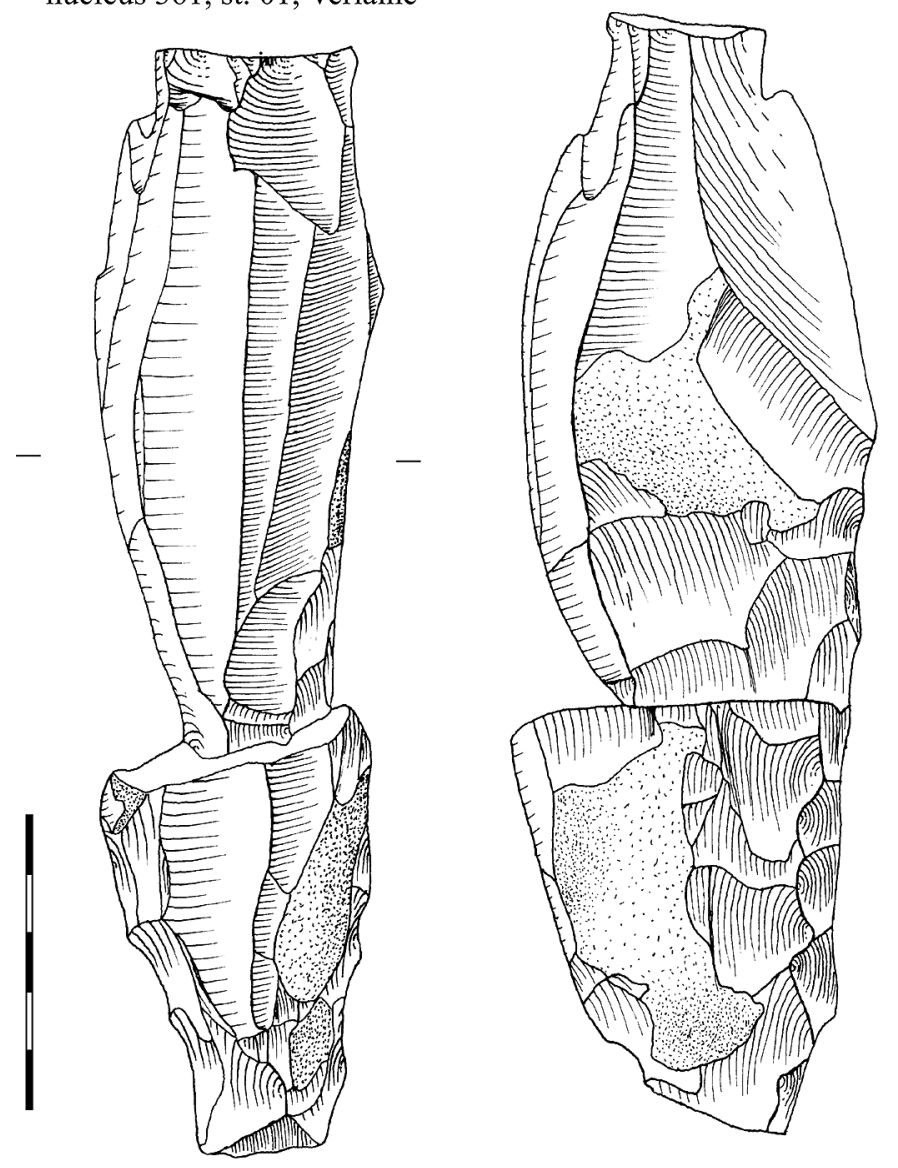

Fig. 3 - Les deux grands nucléus de la structure 01 de Verlaine (Belgique). Fig. 3 - The two big cores of the feature 01 of Verlaine (Belgium). 
étapes du débitage, en Belgique, l'exploitation massive s'accompagne d'une variante du débitage des lames assez caractéristique de la région et du Limbourg hollandais (réalisée sur un autre matériau, le silex de Rijckholt). Elle se traduit par des produits normés avec une faible préparation avant le détachement, des talons lisses à corniche partiellement abattue et surplombante, des tablettes massives, des nucléus unipolaires semi tournant à plan de frappe lisse. Cette méthode se retrouve aussi bien dans les amas que dans les fosses latérales des maisons.

Une autre variante, bien identifiée dans le RRBP du Bassin parisien est probablement la plus commune dans le Rubané occidental mais la moins évidente à documenter en raison de l'absence d'ensemble remonté. Elle se caractérise par un facettage systématique du plan de frappe avant le détachement et un débitage tournant. Une gestion raisonnée et économique des blocs sur des matériaux le plus souvent régionaux, donc peu faciles d'accès immédiat, semblait être la solution la plus simple dans la plupart des cas pour expliquer cette seconde variante. Néanmoins, on la retrouve également dans des contextes riches en matériaux siliceux comme c'est le cas de la vallée de l'Yonne ou de la Seine, si bien qu'elle semble plutôt répondre à une réelle manière de faire plus qu'à une adaptation économique de ressources lointaines.

Pour en revenir à une comparaison au niveau des savoir faire engagés, rien ne permet de supposer une différence entre ces deux variantes, elle s'oppose plutôt dans leur exécution : celle des amas belgo-hollandais est de toute évidence d'exécution plus rapide avec des préparations simplifiées au détachement des lames et des surfaces laminaires moins étendues, souvent frontal et donc plus simple également. On notera une moins bonne régularité générale des produits hesbignons que ceux que l'on rencontre dans les ensembles du Bassin parisien ou dans le Hainaut, mais c'est un critère qualitatif peu évident à mettre en évidence, sauf avec l'emploi d'un codage excessif des paramètres de chacun des produits laminaires. Néanmoins, la variante bien documentée dans les amas peut être interprétée comme le résultat d'une volonté de production rapide et serait donc en rapport avec ce phénomène économique.

\section{PRODUCTION SPÉCIALISÉE ET SPÉCIALISTE}

Nous avons présenté ici les différents éléments dans l'industrie de la pierre taillée qui sont à notre avis les plus convaincants pour aborder la question de la spécialisation des activités pour le Rubané. Il semble évident que deux aspects retiennent particulièrement l'attention et permettent d'avancer plusieurs hypothèses sur le caractère spécialisé d'une partie de la chaîne opératoire de taille. Le premier concerne l'extraction des blocs de silex puisque nous l'avons vu, la gestion des ressources siliceuses pour cette période est orientée vers des sources de bonne qualité qui ne sont pas disponibles partout. On connaît vraisemblablement des centres miniers pour le Rubané, du moins d'après les dates ${ }^{14} \mathrm{C}$, qui sont qualifiés comme participant d'activités spécialisées ou effectués par spécialistes pour les auteurs (Lech, 2003). Néanmoins, cet aspect ne sera pas abordé ici car le phénomène minier nous semble encore mal caractérisé pour cette période, il semble plus judicieux de le supposer dans l'attente de données plus solides. Par ailleurs, rien ne démontre que cette activité soit réellement spécialisée pour cette période non plus. Elle participe de l'acquisition des ressources mais tant que les modalités ne sont pas mieux définies, il nous semble difficile de l'intégrer dans ce débat.

La question de la spécialisation se pose clairement en ce qui concerne le phénomène des productions intensives qui sont observées dans les amas. Si on résume les différents arguments, nous avons les vestiges d'une production massive qui se distingue des activités domestiques habituelles, d'après la composition des vestiges et le type de rejet rapide que l'on retrouve dans toute sorte de structure; y compris les fosses latérales mais sous une forme de rejet distinct des couches détritiques habituelles de ces fosses (Allard et BurnezLanotte, à paraître). Cette production est de plus le résultat de tailleurs expérimentés et elle est orientée vers des produits normés destinés à être mis en circulation dans des réseaux de distribution de silex qui approvisionnent des populations parfois distantes de centaines de kilomètres.

La notion de spécialisation a fait l'objet d'une littérature importante, mais plusieurs travaux sont communément acceptés comme base de discussion (notamment : Costin, 1991 et 2001; Rice, 1991). On peut extraire des critères communs dans ces études qui servent de base à la définition de la spécialisation.

La quantité d'objets produits et la productivité sont des facteurs importants par rapport à ce qui semble être la norme pour une période. En second, le degré de standardisation de la production ou de l'activité.

La notion de savoir-faire spécifique qui est souvent associée au degré d'investissement technique dans un type de production est également un facteur participant de la définition. Il implique un niveau de performance plus élevé que celui reconnu dans les productions ordinaires et pose la question de la qualification des acteurs de la production et notamment de l'existence de véritables spécialistes qui manifestent des capacités techniques exclusives. À ce facteur est souvent associée la question de la quotité du temps de travail afin de déterminer si l'on a affaire à des spécialistes ou artisans à temps complet ou à temps partiel.

Enfin l'organisation spatiale de l'activité doit également être bien caractérisée. Il faut distinguer si elle est concentrée ou dispersée, selon différentes échelles d'observation: domestique, villageoise, dans des centres de production spécifiques et à quelle échelle. Ces différents facteurs ne sont pas les seuls mais nous les retiendrons ici car ils apparaissent comme les mieux adaptés pour le Rubané et le type de données archéologiques dont nous disposons.

Au regard de ces différents facteurs qui servent de référence à notion de spécialisation, on peut logiquement dire que le débitage des lames en silex dans les amas est une production spécialisée. En effet, elle 
associe à la fois des critères quantitatifs (production massive supérieure aux besoins de la communauté qui la produit et recherche de la productivté) et des critères qualitatifs évidents (production réalisée par des tailleurs expérimentés). On peut également mettre en avant que l'organisation spatiale semble distincte des activités domestiques. À Darion, l'idée de la sectorisation spatiale des activités est même proposée en opposant un quartier domestique à un quartier «industriel» (Cahen, 1985). Néanmoins, cela repose sur une interprétation du plan du village peu convaincante et qui ne se retrouve pas dans les autres sites connus pour le moment. En revanche, on peut également avancer l'hypothèse que ces sites ne sont pas isolés, mais concentrés dans certaines régions où les sources siliceuses abondent comme sur la commune de Verlaine. Enfin, 1'objectif transparait clairement car ces productions sont destinées à être mises en circulation dans des réseaux de distribution qui peuvent entrer en concurrence (Zimmermann, 1995; Allard, 2005). On reconnaît donc dans chacune des étapes de cette activité les éléments qui composent une production spécialisée.

La question des acteurs de la production et de leur qualification nous semble en revanche plus délicate à trancher et il faut considérer d'une part les savoir-faire engagés, mais également la nature de la production. Il n'existe pas réellement de définition claire concernant les spécialistes des productions simples, c'est d'ailleurs tout l'enjeu du problème qui nous concerne. En effet, dans les exemples les plus explicites pour la Préhistoire et surtout pour l'industrie lithique, les spécialistes sont bien reconnus, mais pour des productions très particulières. On s'est référé au cadre proposé par Jacques Pelegrin pour répondre à cette question : les tailleurs des amas étaient-ils des spécialistes?

Dans une étude récente, cet auteur distingue les productions exceptionnelles du Paléolithique de celles du Néolithique en proposant de réserver le terme de spécialistes pour certaines productions du Néolithique (Pelegrin, 2007). L'argumentation est détaillée en plusieurs temps. Il s'agit d'objets qui présentent une distinction nette avec la production domestique, même lorsque cette dernière peut être d'une destination fonctionnelle proche (opposition entre très grandes lames ou haches, et lames et haches ordinaires). Cette différence repose non seulement sur des caractères morphométriques, mais également sur une réelle distinction technique combinant compétences et techniques particulières (comme la production de lames sur nucléus en «livre de beurre» du Grand-Pressigny). Il est remarqué que ces objets ont parfois une valeur extraordinaire puisqu'on les retrouve parfois dans des contextes singuliers en dépôt ou en contexte funéraire. Enfin, la dimension économique est soulignée puisque la production est souvent concentrée dans quelques centres qui diffusent les objets parfois sur de très grandes distances. En conclusion, le terme de spécialiste est réservé à ceux qui produisent plus que pour leur consommation et/ou des objets singuliers dont ils étaient les seuls à en maîtriser les compétences et le savoir-faire technique.
En ce qui concerne la période danubienne, l'hypothèse de spécialistes de la taille a été avancée pour les tailleurs des grandes lames en silex bartonien du groupe de Blicquy/Villeneuse-Saint-Germain, soit la seconde étape du Néolithique ancien dans notre région. L'argumentation développée sans entrer dans le détail tient à l'exploitation d'un silex particulier pour en tirer des grandes lames $(15-20 \mathrm{~cm})$ qui se distinguent de la production domestique d'une part par des dimensions plus élevées et d'autre part, par une compétence des tailleurs plus important, qui ont mis en jeu des préparations complexes à triple crête notamment. Ces productions sont l'apanage de certains sites qualifiés de «producteurs » et ces produits ont fait l'objet d'un réseau de distribution spécifique entre le nord du Bassin parisien et la Belgique (Bostyn, 1994 et 1997).

Pour le Rubané, la question s'oriente principalement vers une comparaison entre les structures domestiques (les fosses latérales des unités d'habitation) et les amas de débitage (qui sont, rappelons le, des rejets secondaires dans diverses structures). Concernant les amas, les remontages ont permis d'évaluer les connaissances des tailleurs. Il apparaît que ce sont des bons tailleurs qui ont exécutés les débitages et qu'il n'existe pas, dans le registre des modalités, les éléments flagrants d'une différence dans les connaissances des tailleurs en présence. La gestion complexe de certains blocs pose clairement la question de savoir s'ils sont l'œuvre de tailleurs plus expérimentés que les autres, c'est-à-dire si nous ne sommes pas en présence de différents degrés de compétence au sein d'un très bon niveau de taille. À Verlaine, les divergences révèlent plutôt la présence de plusieurs mains qui sont d'un excellent niveau de taille. Au titre des divergences, on peut remarquer la présence de quelques rares nucléus d'une taille exceptionnelle ou encore le rythme du débitage qui diverge entre les amas (l'amas st. 01 montre une prédominance du code 212 , tandis que celui de la fosse 131 présente un code 123). L'amas de Liège présente de toute évidence des débitages plus simples, mais bien exécutés. Des études plus fines seraient nécessaires, mais on peut raisonnablement admettre que ces différences sont le fruit de différentes mains.

La comparaison avec le mobilier des structures domestiques prend tout son intérêt sur les sites qui livrent des amas et Verlaine fera office de référence dans l'attente d'autres études qui font défauts jusqu'à présent. La comparaison technologique (Allard, 2005 p. 130-153) montre que les assemblages domestiques se différencient par l'apparition de l'outillage (on peut remarquer la forte fréquence des nucléus repris en percuteurs), des éclats d'utilisation et des pièces brûlées. Ces différents objets n'existent pas ou pratiquement pas dans l'amas. Les déchets des toutes premières étapes de la mise en forme des blocs sont également peu présents dans les structures domestiques. En revanche, aucune différence qualitative n'a pu être perçue en ce qui concerne la méthode de taille ou la production. Pour tous les critères directement comparables comme la préparation aux détachements des lames ou encore le gabarit des produits laminaires, les critères sont identiques. L'impression générale est 
que d'une part, la taille du silex a été réalisée partout à Verlaine et toutes les structures livrent du mobilier lithique sous différentes formes de rejet et d'autre part, rien n'oppose clairement les tailleurs des amas des tailleurs des unités domestiques.

La question des spécialistes perd de sa pertinence dans ce cas à notre avis car l'argumentation devient subjective et surtout ne permet pas d'établir des critères clairs pour une définition acceptable. On imagine très bien que la production massive de lames a été réalisée par les meilleurs tailleurs qui étaient probablement reconnus pour leur compétence. Néanmoins, le mobilier des maisonnées ne se distingue pas si bien qu'il est parfaitement acceptable que ce soit les mêmes tailleurs qui réalisaient la production domestique. C'est un fait que les maisons rubanées apparaissent autonomes du point de vue de leur production. De plus, il est parfaitement clair que la production des amas et similaire à celle des unités domestiques. C'est un facteur crucial dans la réflexion qui nous conduit à proposer que la distinction efficace entre spécialiste et non spécialiste doit s'appuyer dans ce cadre sur cet argument. C'est toute la différence avec la seconde étape du Néolithique ancien. Le débitage des grandes lames en silex bartonien ou en silex de Ghlin se distingue très nettement des productions domestiques habituelles et reconnues dans toutes les maisonnées (Bostyn, 1994 et 2007). Ainsi, entre ces deux périodes, ces productions bien exécutées et dont les produits intègrent des réseaux de circulation, on serait à même de réserver le terme de spécialiste pour les tailleurs des grandes lames du Blicquy/Villeneuve-Saint-Germain et de parler plutôt d'experts pour les tailleurs rubanés.

\section{CONCLUSION}

La chaîne opératoire de la taille du silex des premières populations néolithiques de l'Europe tempérée montre une organisation économique selon un système double. D'une part, on observe une grande autonomie des maisonnées dans la taille du silex mais d'autre part, il existe de véritables réseaux de distribution de silex qui approvisionnent des habitats où les ressources sont peu abondantes. La question de la spécialisation se pose clairement à propos d'une partie de ces réseaux de circulation de lames en silex. Nous avons pu voir que l'acquisition de la matière première et le débitage des lames sont les deux éléments qui indiquent l'existence d'une production que nous qualifions de spécialisée. En effet, ces deux étapes associent des arguments quantitatifs et qualitatifs qui s'intègrent bien dans les définitions utilisées pour cette notion. Si les modalités de l'acquisition sont encore en grande partie à définir pour la plupart des régions, l'existence de minière à silex et la quantité de blocs exploités suggèrent largement d'y voir une acquisition intensive des ressources siliceuses. Le débitage des lames que l'on retrouve dans les amas de Hesbaye fait clairement l'objet d'une recherche de production optimale réalisée par des très bons tailleurs. Les déchets de cette production qui concerne des milliers de lames ou des dizaines de milliers de lames comme on peut le supposer, ne se retrouvent pas dans les couches détritiques des fosses latérales des unités d'habitation. Ce phénomène est donc déconnecté des activités habituelles de l'unité domestique. Enfin, il transparait également que cette production est excédentaire par rapport aux besoins de la communauté. C'est donc ce cumul d'arguments qui nous conduit à proposer que la production de lames en silex que l'on perçoit dans ces réseaux est spécialisée.

En revanche, concernant la question du statut des tailleurs, cela tient essentiellement à un problème de définition. Le mobilier des amas de débitage montre clairement que les blocs ont été débités par de bons tailleurs ou d'excellents tailleurs. Néanmoins, ces débitages montrent également que les lames débitées ne différent pas de la production domestique. Les modules sont identiques et correspondent à la norme en vigeur pour cette période, qui est assez stricte. Il s'agit de petites lames en silex de 8 à $12 \mathrm{~cm}$ de long. De même, dans l'état de nos connaissances, rien au final ne permet de faire une réelle différence qualitative entre les débitages des amas et la production domestique. Cela semble au contraire très homogène et seuls des remontages fins permettraient peut être d'y voir une différence; mais pour le moment les méhodes et les procédés de débitage et les lames produites sont identiques. L'amas du site de Liège «Place Saint-Lambert» (Cahen, 1984) présente un mobilier avec des préparations simples qui est tout à fait caractéristique de ce qu'on retrouve dans les unités domestiques du Rubané. La seule différence évidente est à Verlaine la présence d'une volonté de productivité optimale pour les grands blocs dans les amas, mais cela correspond plutôt à une gestion économique liée à cette activité.

Au final, il nous semble plus judicieux de garder le terme de spécialiste à des personnes qui produisent des biens qualitativement distincts de ceux de la sphère domestique ou pour des productions différentes de celles que tout le monde est capable de faire. Cette distinction nous semble utile pour proposer un cadre pour la définition du spécialiste pour la Préhistoire récente, car elle offre une limite qui se base sur des éléments tangibles pour nos contextes archéologiques déjà très restreints.

\section{RÉFÉRENCES BIBLIOGRAPHIQUES}

\footnotetext{
ALLARD P. (2005) - L'industrie lithique des populations rubanées du Nord-Est de la France et de la Belgique, Espelkamp, Marie Leidorf (Internationale Archäologie, 86), 242 p.
}

ALLARD P. (2007) - Surplus Production of Flint Blades in the Early Neolithic of Western Europe: New Evidence from Belgium, European Journal of Archaeology, 8 (2005), p. 205-223. 
ALLARD P., BURNEZ-LANOTTE L. (2008) - An Economy of Surplus Production in the Early Neolithic of Hesbaye (Belgium): Bandkeramik Blade Debitage at Verlaine "Petit Paradis", in P. Allard, F. Bostyn, F. Giligny et J. Lech (dir.), Flint Mining in Prehistoric Europe: Interpreting the Archaeological Records, session tenue au XII ${ }^{\mathrm{e}}$ Congrès de l'European Association of Archaeologists (Cracovie, 19-25 septembre 2006), Oxford, Archaeopress, (BAR, International Series 1891), p. 3139.

ALLARD P., BURNEZ-LANOTTE L. (à paraitre) - Relations stratigraphiques, rapports chronologiques et spatialisation des activités domestiques : étude de cas dans le village producteur de lames du «Petit Paradis» à Verlaine (Hesbaye, Belgique), in P. Allard, C. Hamon et M. Ilett (éd.), The Domestic Space in LBK Settlements, actes de la table ronde internationale (Paris, 7-8 octobre 2010).

ALLARD P., AUGEREAU A., BEUGNIER V., BURNEZ-LANOTTE L., BOSTYN F., CASPAR J.-P., GILIGNY F., HAMARD D., MARTIAL E., PHILIBERT S. (2004) - Fonction des outillages lithiques dans le Bassin parisien au Néolithique, in P. Bodu et C. Constantin (dir.), Approches fonctionnelles en Préhistoire, actes du XXVE Congrès préhistorique de France (Nanterre, 24-26 novembre 2000), Paris, Société préhistorique française, p. 181-192.

ALLARD P., HAMON C., SIDÉRA I. (2007) - Investissement technique et élaboration des productions lithiques et osseuses du Rubané du Bassin parisien, in L. Astruc (dir.), Normes techniques et pratiques sociales. De la simplicité des outillages pré- et protohistoriques, actes des XXVI ${ }^{\text {es }}$ Rencontres internationales d'archéologie et d'histoire (Antibes, 20-22 octobre 2005), Juan-les-Pins, ACPDA, p. 185-194.

BAKELS C. (1982) - The Settlement System of the Dutch Linearbandkeramik, Analecta Praehistorica Leidensia, 15, p. 31-44.

BOSTYN F. (1994) - Caractérisation des productions et de la diffusion des industries lithiques du groupe Néolithique du Villeneuve-SaintGermain, thèse de doctorat, université Paris X - Nanterre, 2 vol., $745 \mathrm{p}$.

BOSTYN F. (1997) - Characterization of Flint Production and Distribution of the Tabular Bartonian Flint during the Early Neolithic (Villeneuve-Saint-Germain Period) in France, in R. Schild et Z. Sulgostowska (dir.), Man and Flint, actes du $7^{\mathrm{e}}$ International Flint Symposium (Varsovie, septembre 1995), Varsovie, Institute of Archaeology and Ethnology, Polish Academy of Sciences, p. 171-183.

BURNEZ-LANOTTE L., ALLARD P. (2003) - Blade Debitage in the Belgium Linearbandkeramik: the Production at Harduémont "Petit Paradis" (Verlaine), in L. Burnez-Lanotte (dir.), Production and Management of Lithic Materials in the European Linearbandkeramik, actes du $15^{\mathrm{e}}$ Congrès de l'UISPP (Liège, septembre 2001), Oxford, Archaeopress (BAR, IS 1200), p. 59-64.

CAHEN D. (1984) - Technologie du débitage laminaire, in M. Otte (dir.), Les fouilles de la place Saint-Lambert à Liège, vol. 1, Liège, université de Liège (ERAUL, 18), p. 171-198.

CAHEN D. (1985) - Interprétations nouvelles du site de Darion, Bulletin de la Société royale belge d'anthropologie et de Préhistoire, 96, p. $75-86$

CASPAR J.-P. (1988) - Contribution à la tracéologie de l'industrie lithique du Néolithique ancien dans l'Europe nord-occidentale, thèse de doctorat, université de Louvain, 3 vol.

COSTIN C. L. (1991) - Craft Specialization: Issues in Defining, Documenting, and Explaining the Organization of Production, in M. B. Schiffer (dir.), Archaeological Method and Theory, vol. 1, Tucson, University of Arizona Press, p. 1-56.

COSTIN C. L. (2001) - Craft Production Systems, in G. Feinman et T. Price (dir.) Archaeology at the Millennium: A Sourcebook, New York, Kluwar Academic et Plenum Press, p. 273-327.

DE GROOTH M. E. T. (1987) - The Organisation of Flint Tool Manufacture in the Dutch Bandkeramik, Analecta Praehistorica Leidensia, 20 , p. $27-52$.
JADIN I. (1990) - Économie de production dans le Rubané récent de Belgique. Approche comparative des industries lithiques de trois villages, in D. Cahen et M. Otte (dir.), Rubané et Cardial, actes du colloque (Liège, novembre 1988), Liège, université de Liège (ERAUL, 39), p. 147-154.

LECH J. (1987) - Danubian Raw Material Distribution Patterns in Eastern Central Europe, in G. De Sievering et M. H. Newcomer (éd.), The Human Uses of Flint and Chert, actes du $4^{\mathrm{e}}$ International Flint Symposium (Brighton, 10-15 avril 1983), Cambridge et New York, Cambridge University Press, p. 241-248.

LECH J. (1990) - The Organization of Siliceous Rock Supplies to the Danubian Early Farming Communities (LBK): Central European Examples, in D. Cahen et M. Otte (éd.), Rubané et Cardial, actes du colloque (Liège, novembre 1988), Liège, université de Liège (ERAUL, 39), p. 51-59.

LECH J. (2003) - Mining and Siliceous Rock Supply to the Danubian Early Farming Communities (LBK) in the Eastern Central Europe: a Second Approach, in L. Burnez-Lanotte (éd.), Production and Management of Lithic Materials in the European Linearbandkeramik, actes du $15^{\circ}$ Congrès de 1'UISPP (Liège, septembre 2001), Oxord, Archaeopress (BAR, IS 1200), p. 59-64.

MAUVILLY M. (1997) - L'industrie lithique de la culture à céramique linéaire de haute et de basse Alsace : état des recherches et bilan provisoire, in C. Jeunesse (éd.), Le Néolithique danubien et ses marges entre Rhin et Seine, actes du $22^{\mathrm{e}}$ Colloque interrégional sur le Néolithique (Strasbourg, 27-29 octobre 1995), Strasbourg, Association pour la promotion de la recherche archéologique en Alsace, p. 327358.

PELEGRIN J. (2007) - Réflexions sur la notion de «spécialiste» dans la taille de la pierre au Paléolithique, in R. Desbrosses et A. Thévenin (dir.), Des grottes de Châtelperron au musée des Antiquités nationales : hommages à Henri Delporte, Paris, CTHS (Documents préhistoriques, 24), p. 315-318.

RICE P. M. (1991) - Specialization, Standardization, and Diversity: a Retrospective, in R. L. Bishop et F. W. Lange (dir.), The Ceramic Legacy of Anna O. Shepard, Boulder (Co), University Press of Colorado, p. 257-279.

SOMMER U. (2006) - The Linearbandkeramik Settlement of HanauKlein Auheim (Hesse): a specilialised Mining Settlement?, in G. Kłorlin et G. Weisgerbe (éd.), Stone Age, Mining Age, actes du $8^{\mathrm{e}}$ International Flint Symposium (Bochum, septembre 1999), Bochum, Bergbau Museum (Veröffentlichungen aus dem Deutschen Berghau-Museum Bochum, 148; Der Anschnitt: Zeitschrift für Kunst and Kultur im Bergbau, 19), p. 187-194.

VAN GIJN A. (1990) - The Wear and Tear of Flint. Principles of Functional Analysis Applied to Dutch Neolithic Assemblages, Leyde, University of Leiden (Analecta Praehistorica Leidensia, 22), 182 p.

VAUGHAN P. (1994) - Microwear Analysis of Flints from the Bandkeramik Sites of Langweiller 8 and Laurenzberg 7, in J. Lüning et P. Stehli (dir.), Die Bandkeramik im Merzbachtal auf der Aldenhovener Platte, Cologne, Rheinland Verlag (Rheinische Ausgrabungen, 36), p. 535-552.

ZIMMERMANN A. (1995) - Austauschsysteme von Silexartefakten in der Bandkeramik Mitteleuropas, Bonn, Habelt (Universitätsforschungen zur prähistorischen archäologie, 37), $162 \mathrm{p}$.

\section{Pierre ALLARD}

CNRS, UMR 7055 «Préhistoire et technologie» Maison de l'archéologie et de l'ethnologie René-Ginouvès

21, allée de l'Université, 92023 Nanterre cedex pierre.allard@mae.u-paris10.fr 